\title{
Article \\ White Matter Microstructural Alterations in Newly Diagnosed Parkinson's Disease: A Whole-Brain Analysis Using dMRI
}

\author{
Jun-Yeop Kim ${ }^{1}{ }^{\circledR}$, Jae-Hyuk Shim ${ }^{2}$ and Hyeon-Man Baek ${ }^{1,2, *}$ \\ 1 College of Medicine, Gachon University, Incheon 21565, Korea; luckyjunyeop10@gmail.com \\ 2 Department of Health Sciences and Technology, GAIHST, Gachon University, Incheon 21999, Korea; \\ jaehyukshim11@gmail.com \\ * Correspondence: hmbaek98@gachon.ac.kr; Tel.: +82-010-9878-4279
}

Citation: Kim, J.-Y.; Shim, J.-H.; Baek, H.-M. White Matter

Microstructural Alterations in Newly

Diagnosed Parkinson's Disease: A

Whole-Brain Analysis Using dMRI Brain Sci. 2022, 12, 227. https:/ / doi.org/10.3390/brainsci12020227

Received: 10 December 2021

Accepted: 3 February 2022

Published: 7 February 2022

Publisher's Note: MDPI stays neutral with regard to jurisdictional claims in published maps and institutional affiliations.

Copyright: (C) 2022 by the authors. Licensee MDPI, Basel, Switzerland. This article is an open access article distributed under the terms and conditions of the Creative Commons Attribution (CC BY) license (https:// creativecommons.org/licenses/by/ $4.0 /)$.

\begin{abstract}
Parkinson's disease (PD) is a neurodegenerative disorder characterized by cardinal motor symptoms and other non-motor symptoms. Studies have investigated various brain areas in PD by detecting white matter alterations using diffusion magnetic resonance imaging processing techniques, which can produce diffusion metrics such as fractional anisotropy and quantitative anisotropy. In this study, we compared the quantitative anisotropy of whole brain regions throughout the subcortical and cortical areas between newly diagnosed PD patients and healthy controls. Additionally, we evaluated the correlations between the quantitative anisotropy of each region and respective neuropsychological test scores to identify the areas most affected by each neuropsychological dysfunction in PD. We found significant quantitative anisotropy differences in several subcortical structures such as the basal ganglia, limbic system, and brain stem as well as in cortical structures such as the temporal lobe, occipital lobe, and insular lobe. Additionally, we found that quantitative anisotropy of some subcortical structures such as the basal ganglia, cerebellum, and brain stem showed the highest correlations with motor dysfunction, whereas cortical structures such as the temporal lobe and occipital lobe showed the highest correlations with olfactory dysfunction in PD. Our study also showed evidence regarding potential neural compensation by revealing higher diffusion metric values in early-stage PD than in healthy controls. We anticipate that our results will improve our understanding of PD's pathophysiology.
\end{abstract}

Keywords: Parkinson's disease; whole brain; diffusion MRI; quantitative anisotropy (QA)

\section{Introduction}

Parkinson's disease (PD) is a degenerative disorder characterized by progressive cardinal motor symptoms such as resting tremor, rigidity, bradykinesia, and postural instability. Such symptoms can be traced to the various systematic changes that occur in motor networks due to dopamine depletion, mainly in the substantia nigra pars compacta $(\mathrm{SNpc})[1,2]$. In addition to these motor symptoms, non-motor symptoms (NMS), including olfaction dysfunction, autonomic dysfunction, and REM sleep behavior disorder (RBD), also manifest in PD due to $\alpha$-synuclein accumulation in specific pathways throughout the brain [3]. Many studies have suggested that NMS can be used as a diagnostic marker of prodromal and/or early PD [4,5]. Recently, diffusion magnetic resonance imaging (dMRI) has been used to diagnose early PD by assessing white matter (WM) connectivity in some brain areas related to motor symptoms as well as NMS [6].

$\mathrm{dMRI}$ is a unique tool that can be used to measure WM microstructure in vivo by characterizing the diffusion displacement of water molecules in white matter. Diffusion tensor imaging (DTI) has been used as a common dMRI processing technique to obtain gross fiber orientation of white matter voxels and use them to calculate quantitative diffusion metrics, such as fractional anisotropy (FA) and mean diffusivity (MD) [7]. Various studies have shown correlations between significantly high or low levels of diffusion metrics and 
alterations in WM microstructural integrity, such as demyelination and necrosis [8-10]. However, while DTI has been used in a significant number of studies, it has some limitations such as the inability to resolve complex fiber orientations such as crossing or branching patterns. To overcome such limitations, a novel approach called generalized q-sampling imaging (GQI) was developed. Unlike DTI, which is a model-based method, GQI is a model-free method based on the Fourier transform between diffusion MR signals and the underlying diffusion displacement, providing more precise directional and quantitative information regarding neural structures. Overall, GQI has shown advantages over conventional diffusion tensor approaches due to its higher sensitivity in fiber tracking and wide applicability [11]. GQI provides a new quantitative diffusion metric called quantitative anisotropy (QA), which represents the density of WM from the calculations of water diffusivity in specific directions and has shown better accuracy in reconstructing images compared with other conventional metrics such as FA [12]. Due to the multiple advantages mentioned above, many studies have used GQI to investigate the pathological mechanisms of WM microstructures. For instance, QA alterations showed significant correlations with various pathological changes such as the peritumoral infiltration of gliomas and demyelination in radiation-induced brain injuries $[13,14]$.

Many studies have used dMRI to compare diffusion metrics between PD patients and healthy controls (HCs) to observe whether the degeneration of neuronal microstructure can be observed in the form of significant diffusion metric changes throughout various areas of the brain. Most studies focused on specific brain areas, such as the substantia nigra (SN) and basal ganglia, which are well known to be affected in PD $[15,16]$. While there have been some studies that have investigated multiple brain areas other than the areas mentioned above [17], there is only a small number of studies focusing on the entire brain area using diffusion metrics. Several recent dMRI analysis studies have shifted from using conventional metrics to using QA; however, most of these studies investigated the differences in specific WM tracts rather than in whole brain areas between HCs and PD patients [18-20]. In addition, studies using diffusion metrics, such as FA and QA, have provided conflicting results. Although decreased diffusion metrics have been frequently reported in PD [21-23], there are several occasions where results showed higher FA and QA in PD than in HC, which were commonly interpreted as neural compensation that occur in the early stages of neurodegeneration $[17,24,25]$. In this regard, a systemic review published in 2020 suggested that regions other than the hallmark regions of PD, such as SN, should be investigated more extensively and coherently using advanced dMRI processing techniques [26].

In this study, we extensively compared the QA of multiple brain regions between PD and HC. We compared not only the regions affected directly by dopaminergic neuronal loss, but also some scarcely investigated regions. We delineated various structures and classified them into larger parts such as the basal ganglia, limbic system, cerebellum, thalamus, brain stem, corpus callosum, frontal lobe, parietal lobe, occipital lobe, temporal lobe, and insular lobe. In addition, we analyzed the correlations between the QAs of various brain areas and neuropsychological test scores in PD to determine the impact of WM degeneration in each brain area on several neuropsychological dysfunctions. We anticipate that this study can improve the current understanding of PD neuropathology through dMRI processing of several areas rarely touched upon in PD studies.

\section{Materials and Methods}

\subsection{Participants}

A total of $44 \mathrm{HCs}$ and $44 \mathrm{PD}$ participants in this study were recruited from the Parkinson's Progression Markers Initiative (PPMI) open-access database (www.ppmi-info.org/ data, accessed on 1 September 2021). Each PD patient was assessed for PD using part III of the Movement Disorder Society-sponsored revision of the United Parkinson's Disease Rating Scale (MDS-UPDRS III), dopamine transporter SPECT scans to observe the dopaminergic neurons, and manifested motor symptoms such as bradykinesia and rest- 
ing tremor. Assessment of PD was performed before any patients were administered PD medication, which could interfere with PD symptom testing. All participants in this study tested negative for neurological disorders other than PD. The group demographics and clinical characteristics are shown in Table 1. All participants provided written informed consent to share their unidentified clinical data with the investigators.

Table 1. Group characteristics of PD and HC.

\begin{tabular}{cccc}
\hline Group & HC $(\boldsymbol{n}=\mathbf{4 4 )}$ & PD $(\boldsymbol{n}=\mathbf{4 4})$ & $p$-Value \\
\hline Age (mean \pm SD) & $60.4 \pm 9.6$ & $58.3 \pm 9.3$ & 0.316 \\
Sex (male/female) & $27 / 17$ & $27 / 17$ & - \\
Dominant side (left/right) & - & $23 / 21$ & - \\
Education years (mean \pm SD) & $16.2 \pm 2.9$ & $15.3 \pm 3.1$ & 0.186 \\
Age onset in years (mean \pm SD) & - & $56.6 \pm 9.7$ & - \\
Duration of disease in months (mean \pm SD) & - & $6.1 \pm 6.4$ & - \\
H \& Y scale (mean \pm SD) & $0.0 \pm 0.0$ & $1.5 \pm 0.5$ & $<0.001$ \\
MDS-UPDRS III score (mean \pm SD) & $0.7 \pm 1.1$ & $19.7 \pm 9.1$ & $<0.001$ \\
UPSIT score (mean \pm SD) & $33.3 \pm 4.8$ & $23.7 \pm 6.9$ & $<0.001$ \\
SCOPA-AUT score (mean \pm SD) & $5.4 \pm 2.7$ & $8.7 \pm 5.7$ & $<0.001$ \\
RBDSQ score (mean \pm SD) & $2.6 \pm 2.0$ & $3.6 \pm 2.0$ & 0.017 \\
GDS score (mean \pm SD) & $1.2 \pm 2.5$ & $1.8 \pm 1.7$ & 0.154 \\
MoCA score (mean \pm SD) & $28.4 \pm 1.1$ & $28.0 \pm 1.7$ & 0.188
\end{tabular}

HC, healthy controls; PD, Parkinson's disease; SD, standard deviation; H \& Y, Hoehn and Yahr; MDS-UPDRS III Movement Disorder Society-sponsored revision of the Unified Parkinson's Disease Rating Scale; UPSIT, University of Pennsylvania Smell Identification Test; SCOPA-AUT, Scale for Outcomes in Parkinson's disease-Autonomic RBDSQ, REM Sleep Behavior Disorder Screening Questionnaire; GDS, Global Deterioration Scale; MoCA, Montreal Cognitive Assessment Test Scoring.

\subsection{MRI Data Acquisition}

The MRI data of the HC and PD participants used in this study were obtained from the PPMI database (www.ppmi-info.org/data, accessed on 1 September 2021). Diffusion MRI images were acquired using standard protocols on 3T scanners at approximately 11 PPMI imaging sites. T1-weighted images were acquired using a 3D T1-weighted MPRAGE sequence (echo time $(\mathrm{TE})=90 \mathrm{~ms}$, repetition time $(\mathrm{TR})=2300 \mathrm{~ms}, 1 \mathrm{~mm}^{3}$ resolution), and DTI images were acquired using a 2D single-shot echo-planar DTI sequence $\left(\mathrm{TE}=88 \mathrm{~ms}, \mathrm{TR}=900 \mathrm{~ms}, 2 \mathrm{~mm}^{3}\right.$ resolution, 72 slices, flip angle $=90^{\circ}, 64$ gradient directions, $b$-value $=1000 \mathrm{~s} / \mathrm{mm}^{2}$ ). More details regarding MRI sequence information can be found in the PPMI MRI technical operations manual (www.ppmi-info.org, accessed on 1 September 2021).

\subsection{Image Processing}

Each diffusion-weighted MRI image was preprocessed through a series of MRtrix3 image-processing programs, which involved denoising, Gibbs ringing removal, motion and distortion correction, bias field correction, and resampling to a $1 \mathrm{~mm}^{3}$ isotropic resolution $[27,28]$. The brain structures of each subject were segmented through nonlinear spatial normalization of the FreeSurfer segmentation atlas [29] from the MNI152 template space to each subject's respective space using DSI Studio [11]. The QA diffusion measure of each brain structure was obtained using DSI Studio statistics [11].

\subsection{Classification of Brain Structures}

Each segmented structure was classified into a larger part of the brain. The following are the areas of the brain and the individual structures that comprise them: basal ganglia (caudate, putamen, accumbens area, pallidum, and ventral diencephalon); limbic system (hippocampus, amygdala, cingulate gyrus, orbital frontal gyrus, insular gyrus, parahippocampal gyrus, and subcallosal gyrus); cerebellum, thalamus, brain stem, corpus callosum, frontal lobe (precentral gyrus, superior frontal gyrus, middle frontal gyrus, inferior frontal gyrus, orbital gyrus, rectus gyrus, frontomarginal cortex, and transverse frontopolar cor- 
tex); parietal lobe (postcentral gyrus, superior parietal lobule, inferior parietal lobule, and precuneus); occipital lobe (cuneus, lingual gyrus, fusiform gyrus, superior occipital gyrus, middle occipital gyrus, inferior occipital cortex, and occipital pole); temporal lobe (superior temporal gyrus, middle temporal gyrus, inferior temporal gyrus, and temporal pole); and insular lobe.

\subsection{Statistical Analysis}

All statistical analyses were performed using IBM SPSS Statistics for Windows (version 22.0; IBM Corp., Armonk, NY, USA). Demographic, clinical, and neuropsychological data of the participants were analyzed. Within the PD group, QA values of each structure were compared between the most and least affected side using the paired $t$-test. All QA values of each individual segment of the brain were compared between $\mathrm{HC}$ and PD groups using the independent sample $t$-test. The Benjamini-Hochberg procedure was used to correct type 1 errors for multiple comparisons, and a false discovery rate (FDR) level of 0.005 was applied. Pearson's correlation was used to determine the correlation coefficients between the QA values of each brain region and corresponding neuropsychological test scores (significance at $p<0.05$ ).

\section{Results}

Table 1 presents the demographics and clinical characteristics of the HC and PD participants, which were compared for significant differences using the independent sample $t$-test. The "dominant side" means the side most affected at PD symptom onset, and this information was investigated due to the asymmetric characteristic in most newly diagnosed PD [30]. The following scores showed significant differences $(p<0.05)$ between HC and PD participants: the H \& Y scale, an indicator of PD progression; the MDS-UPDRS III score, an indicator of movement disorders; the UPSIT score, an indicator of olfaction functions; the SCOPA-AUT score, an indicator of autonomic symptoms in PD; and the RBDSQ score, an indicator of sleep disorders. The other indices showed no significant differences $(p>0.05)$ between the HC and PD groups.

The information regarding the segmented brain regions from the PPMI database was classified by anatomical or functional criteria. Figure 1 shows each classified area of the brain, which is the main subject we investigated.

The paired $t$-test was used to compare the QAs of each structure between the most affected and the least affected side. We defined most affected side as the contralateral side of the "dominant side" mentioned above, while the least affected side was defined as the opposite side of the most affected side. As a result of the analysis, no regions showed significant QA differences between the most and the least affected side within PD $(p>0.05)$.

The independent sample $t$-test was used to compare the QAs of subcortical structures between HCs and PD patients, as shown in Table 2. With post FDR correction significance set to 0.005 , PD patients showed significantly higher QAs in the basal ganglia, striatum, limbic system, cerebellum, and brain stem compared to HC individuals. Significances of QA differences were seen in both sides no matter how much the side was affected. Figure 2 shows a graph of the QA differences in the subcortical structures between the HC and PD groups.

The independent sample $t$-test was also used to compare the QAs of cortical structures between the HC and PD groups, as shown in Table 3. With post FDR correction significance set to 0.005 , PD patients had significantly higher QAs in the occipital lobe, temporal lobe, and insular lobe compared to HC individuals. Significances of QA differences were seen in both sides no matter how much the side was affected. Figure 2 shows a graph of the QA differences in the cortical structures between the HC and PD groups. 


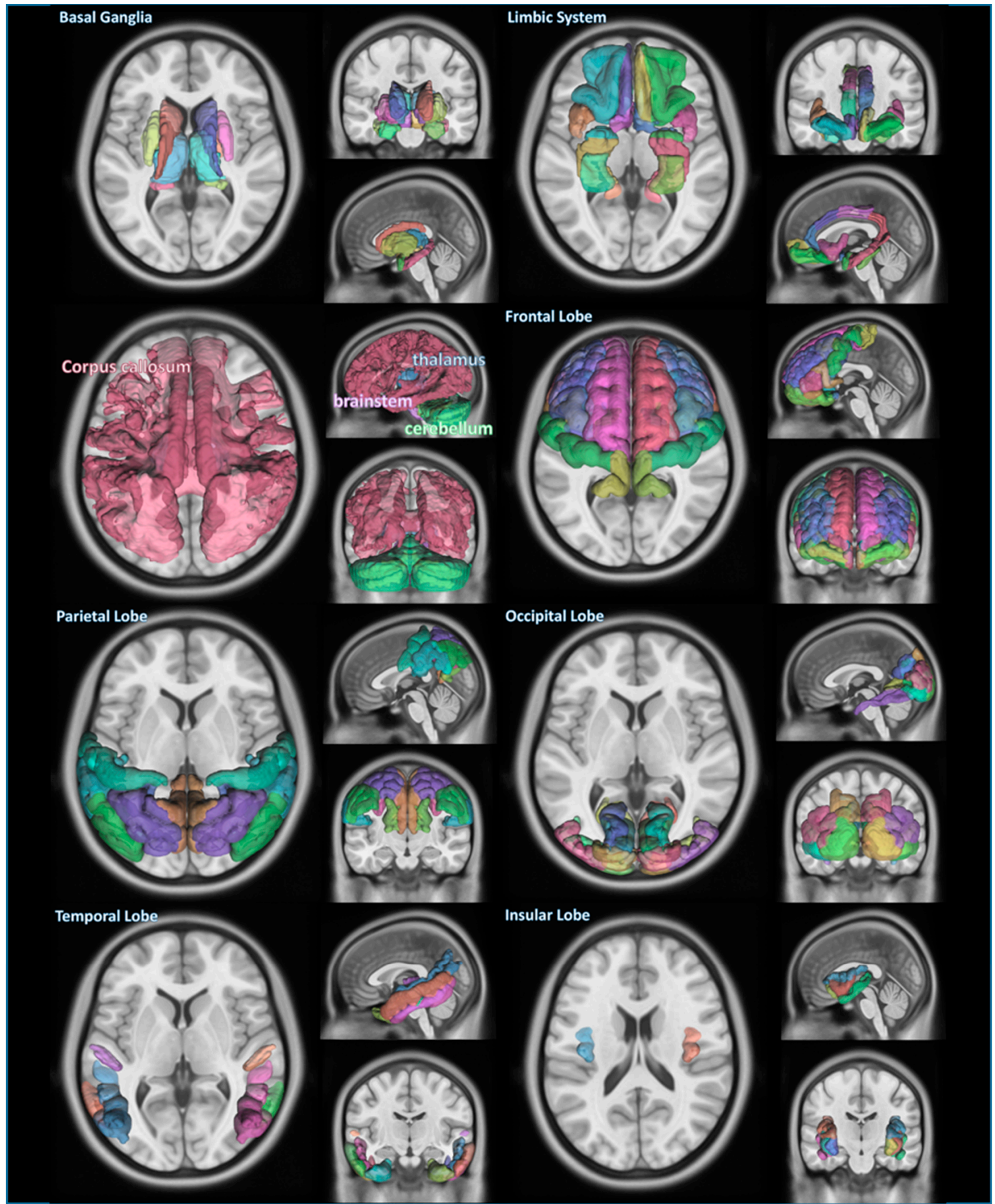

Figure 1. Segmentation of several areas of brain in Parkinson's disease. The areas composed of segmented structures of a PD patient recruited in this study are basal ganglia, limbic system, cerebellum, thalamus, brain stem, corpus callosum, frontal lobe, parietal lobe, occipital lobe, temporal lobe, and insular lobe, which are overlaid on top of MNI templates. 
Table 2. QA differences in the subcortical structures between HC and PD groups.

\begin{tabular}{|c|c|c|c|c|c|}
\hline Region & Subregion & HC QA (Mean \pm SD) & PD QA (Mean \pm SD) & $p 1$ Value & $p 2$ Value \\
\hline \multirow[t]{4}{*}{ Basal ganglia } & & $0.361 \pm 0.031$ & $0.417 \pm 0.103(\mathrm{M})$ & 0.001 & 0.002 \\
\hline & & & $0.418 \pm 0.095(\mathrm{~L})$ & $<0.001$ & $<0.001$ \\
\hline & Striatum & $0.329 \pm 0.030$ & $0.385 \pm 0.086(\mathrm{M})$ & $<0.001$ & $<0.001$ \\
\hline & & & $0.383 \pm 0.093(\mathrm{~L})$ & $<0.001$ & $<0.001$ \\
\hline \multirow[t]{4}{*}{ Limbic system } & & $0.234 \pm 0.020$ & $0.263 \pm 0.054(\mathrm{M})$ & 0.002 & 0.002 \\
\hline & & & $0.263 \pm 0.053(\mathrm{~L})$ & 0.001 & 0.002 \\
\hline & Cingulate gyrus & $0.209 \pm 0.018$ & $0.228 \pm 0.043(\mathrm{M})$ & 0.008 & 0.004 \\
\hline & & & $0.229 \pm 0.047(\mathrm{~L})$ & 0.011 & 0.005 \\
\hline \multirow[t]{2}{*}{ Cerebellum } & & $0.343 \pm 0.029$ & $0.389 \pm 0.094(\mathrm{M})$ & 0.003 & 0.003 \\
\hline & & & $0.386 \pm 0.092(\mathrm{~L})$ & 0.002 & 0.003 \\
\hline \multirow{2}{*}{ Thalamus } & & $0.398 \pm 0.038$ & $0.454 \pm 0.130(\mathrm{M})$ & 0.008 & 0.004 \\
\hline & & & $0.422 \pm 0.111(\mathrm{~L})$ & 0.003 & 0.003 \\
\hline Brain stem & & $0.474 \pm 0.044$ & $0.551 \pm 0.149$ & 0.002 & 0.003 \\
\hline Corpus callosum & & $0.811 \pm 0.081$ & $0.901 \pm 0.193$ & 0.006 & 0.004 \\
\hline
\end{tabular}

Average QA values of the subcortical structures. " $\mathrm{M}$ " and "L" in PD QA section represent the "most affected side" and "least affected side", respectively. Significant differences and $p$-values were calculated using independent sample $t$-tests ( $p 1$ value), with $p$-values adjusted for multiple corrections using FDR correction ( $p 2$ value). Bolded values represent $\mathrm{QAs}$ with significant differences after FDR correction (FDR $=0.005)$. QA: quantitative anisotropy.

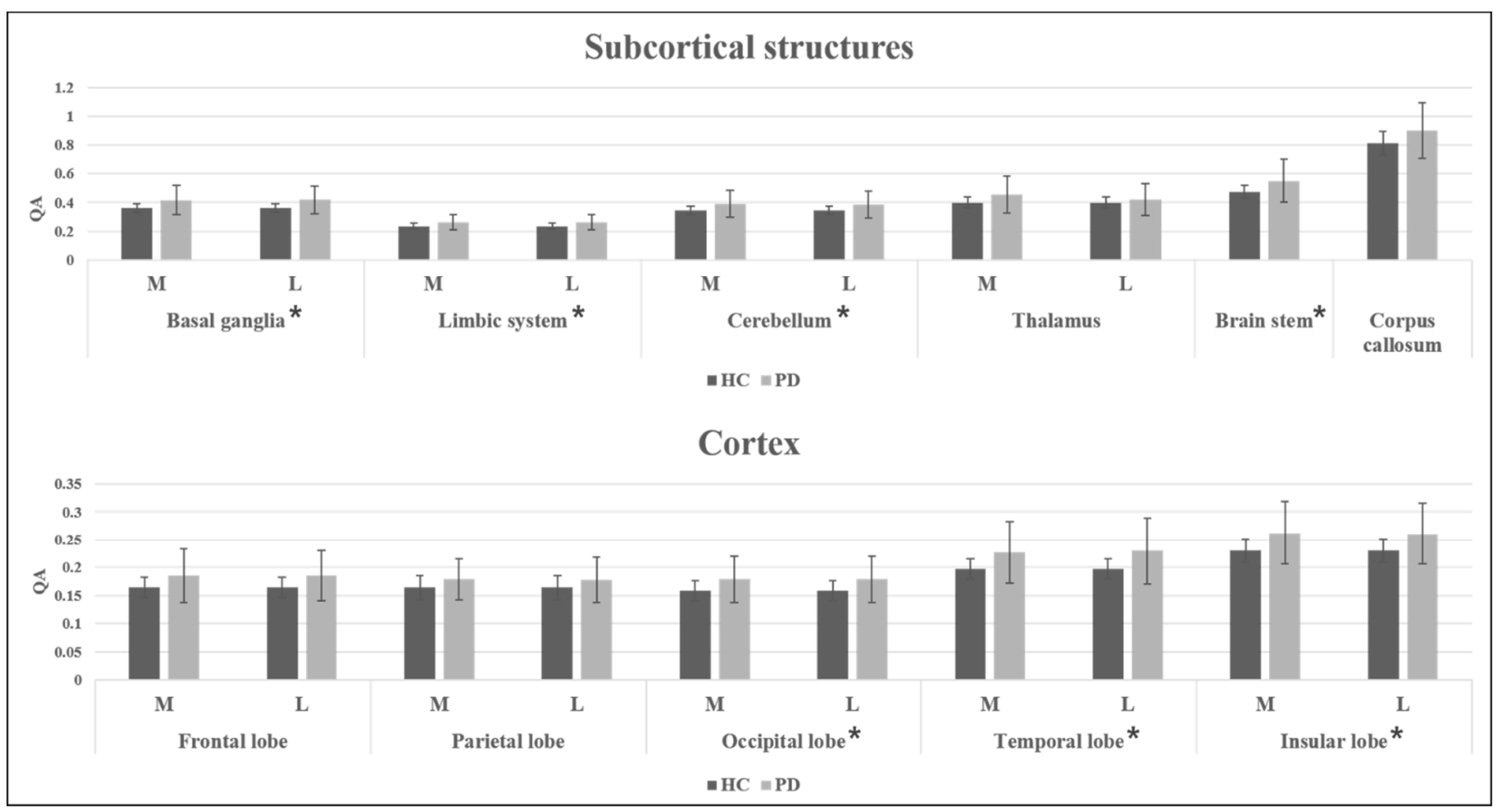

Figure 2. QA differences in subcortical and cortical structures between HC and PD groups. "M" and "L" represent the "most affected side" and "least affected side" in PD. Error bars represent standard errors. Structures with asterisk represent the regions with significant difference after FDR correction.

Correlations between the neuropsychological test (MDS-UPDRS III, UPSIT, SCOPAAUT, and RBDSQ) scores and QAs of each brain region (basal ganglia, limbic system, cerebellum, thalamus, brain stem, corpus callosum, frontal lobe, parietal lobe, occipital lobe, temporal lobe, and insular lobe) were evaluated by calculating correlation coefficients. We conducted this correlation analysis to find out whether each symptom of PD is related to the alterations of $\mathrm{QA}$, a diffusion measure that has been shown to represent WM density in specific brain regions affected in PD. We selected the four neuropsychological tests mentioned above based on the studies that showed motor and some non-motor symptoms 
(olfactory dysfunction, autonomic disturbance, and sleep disturbance) appear in early-stage PD [1,5]. The results of our analysis presented in Table 1 also show significant differences between the HC and PD groups in the four tests mentioned above $(p<0.05)$. Additionally, we conducted an analysis of QA between brain regions of the most affected side and the least affected side and found no significant differences. The results are shown in Figure 3. The MDS-UPDRS III scores were significantly positively correlated with each area mentioned above $(p<0.05)$. Among them, the brain stem $(r=0.399)$, cerebellum $(\mathrm{r}=0.393)$, and basal ganglia $(\mathrm{r}=0.392)$ are the areas which showed the highest correlation coefficients. The UPSIT scores were significantly negatively correlated with every area mentioned above $(p<0.05)$. Among them, the temporal lobe $(r=-0.321)$ and occipital lobe $(\mathrm{r}=-0.313)$ are the areas which showed the highest correlation coefficients in absolute values. The SCOPA-AUT scores were significantly positively correlated with the basal ganglia alone $(r=0.255)$. The RBDSQ showed no significant correlation with any of the regions mentioned above.

Table 3. QA differences in the cortical structures between HC and PD groups.

\begin{tabular}{ccccc}
\hline Region & HC QA (Mean \pm SD) & PD QA (Mean \pm SD) & $p$ 1 Value & $p$ 2 Value \\
\hline Frontal lobe & $0.164 \pm 0.018$ & $0.186 \pm 0.048(\mathrm{M})$ & 0.007 & 0.004 \\
& & $0.186 \pm 0.045(\mathrm{~L})$ & 0.004 & 0.004 \\
Parietal lobe & $0.164 \pm 0.022$ & $0.179 \pm 0.037(\mathrm{M})$ & 0.030 & 0.005 \\
& & $0.178 \pm 0.040(\mathrm{~L})$ & 0.043 & 0.005 \\
Occipital lobe & $0.158 \pm 0.018$ & $0.179 \pm 0.041(\mathrm{M})$ & 0.002 & $\mathbf{0 . 0 0 3}$ \\
& & $0.179 \pm 0.041(\mathrm{~L})$ & 0.003 & $\mathbf{0 . 0 0 3}$ \\
Temporal lobe & $0.197 \pm 0.018$ & $0.227 \pm 0.055(\mathrm{M})$ & 0.001 & $\mathbf{0 . 0 0 1}$ \\
& & $0.230 \pm 0.059(\mathrm{~L})$ & $<0.001$ & $<\mathbf{0 . 0 0 1}$ \\
Insular lobe & $0.231 \pm 0.021$ & $0.262 \pm 0.056(\mathrm{M})$ & $<0.001$ & $\mathbf{0 . 0 0 1}$ \\
& & $0.261 \pm 0.055(\mathrm{~L})$ & 0.002 & $\mathbf{0 . 0 0 2}$
\end{tabular}

Average QA values of cortical structures. "M" and "L" in PD QA section represent the "most affected side" and "least affected side", respectively. Significant differences and $p$-values were calculated using independent sample $t$-tests ( $p 1$ value), with $p$-values adjusted for multiple corrections using FDR correction ( $p 2$ value). Bolded values represent QAs with significant differences after FDR correction (FDR = 0.005). QA: quantitative anisotropy.

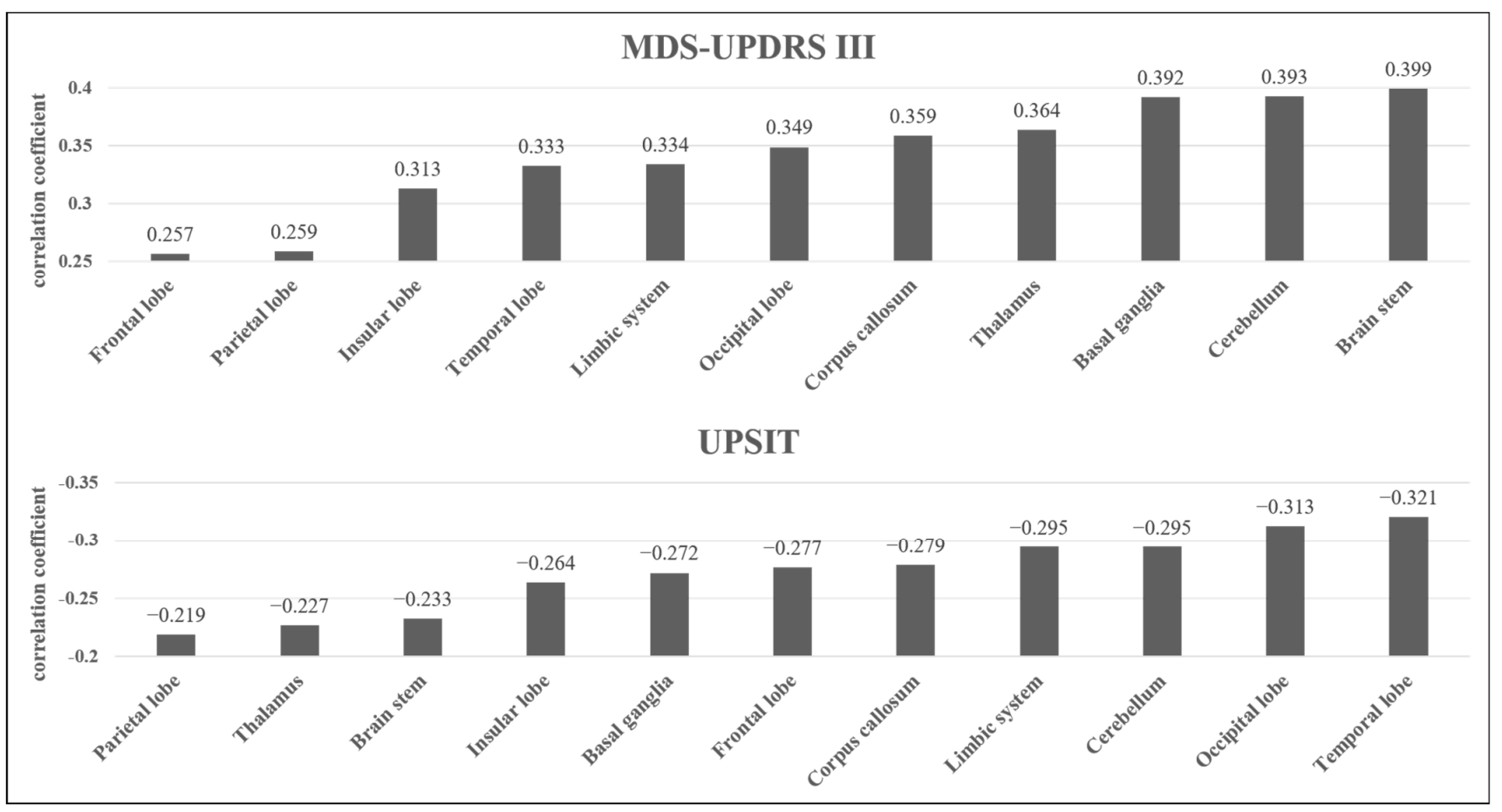

Figure 3. Correlation coefficients of each brain area with MDS-UPDRS III and UPSIT scores. 


\section{Discussion}

In our study, we utilized diffusion MRI images to compare the QAs of the segmented structures throughout the whole brain. These structures included not only specific areas influenced by the dopaminergic neurons, such as the basal ganglia, but also other brain areas that have not been investigated thoroughly using dMRI techniques. Additionally, we investigated the correlations between the QAs of the brain areas and corresponding neuropsychological test scores. Our results revealed that the basal ganglia, limbic system, cerebellum, brain stem in subcortical areas, occipital lobe, temporal lobe, and insular lobe in cortical areas showed significant differences in QAs between the HC and PD groups when the FDR was set to 0.005. In the correlation analysis, the QAs of the brain stem, cerebellum, and basal ganglia showed the highest correlation coefficients with MDSUPDRS III scores, and the QAs of the temporal lobe and occipital lobe showed the highest correlation coefficients in absolute values with UPSIT scores.

A significant number of studies on PD have investigated group differences in diffusion metrics such as FA and QA in brain structures, especially in the basal ganglia, where the death of dopaminergic neurons occurs $[15,16]$. The substantia nigra (SN) is the target structure that has been investigated using various diffusion metrics. However, there have been controversial results in the SN between early PD patients and controls. Some studies found neuronal degeneration and a reduced FA of SN in early PD patients [21,23,31], whereas other studies found no significant differences or even higher values of FA of SN in early PD patients when compared to controls [22,32]. Besides SN, other subcortical structures such as the putamen and cerebellum, which are involved in dopaminergic pathways, have also been investigated. Although there are some conflicting results [17,33], many studies have shown that early PD patients display FA increases in several subcortical areas, such as the putamen $[24,34]$. Recently, many studies have attempted to compare QA values in subcortical areas between HCs and PD patients. Studies have found that prodromal or earlier-stage PD patients showed higher QAs compared to HCs or later-stage PD patients in several WM tracts involved in subcortical areas $[18,25,35]$. These results are in line with the significantly higher QAs in early PD patients compared to HCs that were observed in the specific brain areas considered in our study, including the basal ganglia, cerebellum, and brain stem. These results could provide evidence of compensatory brain plasticity in early PD. Compensatory brain plasticity or neural compensation is a term that explains the dissociation between brain pathology and behavioral alterations occurring in prodromal or early stages of neurodegeneration, such as Alzheimer's disease [36]. Our findings strengthen the hypothesis of compensatory brain plasticity in very early phase PD, as mentioned in previous studies [18,25].

Although subcortical areas have been the target in most studies using DTI, some studies have attempted to find significant results in cortical areas in PD. Structures related to olfaction have often been chosen as the investigation target in PD because olfactory dysfunction is one of the earliest clinical symptoms of PD, even preceding motor symptoms [37]. The olfactory system is an area that includes the piriform cortex and parahippocampal gyrus; it also connects to various areas, including the limbic system structures (amygdala and hippocampus) [38,39]. Previous studies have found differences in FA and QA in the areas or tracts related to the olfactory system between HCs and PD patients [18,35,40]. Our results were similar to those of previous studies in that we showed significantly higher QA values in the temporal lobe, where the olfactory cortex is located, and in the insular lobe, which is known to have a specific role in olfactory processing [41], in early PD patients compared to HCs. Our study also found a difference in QA between the HC and PD groups in the occipital lobe. This could be the result of connectivity alterations in some WM tracts such as the fronto-occipital fasciculus or longitudinal fasciculus, as shown in previous studies [20]. Otherwise, this might be the result of alterations in WM distribution in specific occipital regions such as the optic radiation [19,42], which could be related to visual dysfunction in early PD patients [43]. However, further studies are required to interpret this result more precisely. Higher QAs in PD patients compared to HCs are thought to 
be due to neural compensation appearing in these regions in the early stage of PD [18,25]. QAs of the frontal lobe and parietal lobe showed no significant differences between the $\mathrm{HC}$ and PD groups, which might be the result of only selecting early-stage PD cases as the study subjects, in whom widespread microstructural changes do not occur $[25,31,44]$.

Motor deficits are typical symptoms of PD due to the degeneration of nigrostriatal tracts, which have been shown in many DTI-derived metrics [45]. In our study, higher correlation coefficients between the QAs of the basal ganglia, cerebellum, and brain stem and MDS-UPDRS III scores showed that the microstructure of the regions where nigrostriatal tracts pass through or are well known to be related to motor function were altered the most, and these changes contribute to motor dysfunction in early-stage PD. Olfactory dysfunction is a typical early non-motor symptom in PD [36] and is known to be related to the olfactory system, which is located in the temporal lobes [32,39,46]. In line with this knowledge, the QAs of the temporal lobes showed higher correlations with the UPSIT scores than other regions. This result confirms the previous result that pathological changes in areas of the olfactory system other than the olfactory bulb are in charge of olfactory dysfunction in PD [37]. In addition, the result of a lower correlation coefficient in areas where the nigrostriatal tract passes with UPSIT scores could be interpreted using previous results which revealed that odor identification deficits in PD are not explained by nigrostriatal dopaminergic denervation $[47,48]$. The QAs of the occipital lobes also showed higher correlations with the UPSIT scores. A previous study that investigated the association between olfactory dysfunction and brain microstructures in prodromal PD showed that some fibers, such as the fronto-occipital fasciculus and longitudinal fasciculus, which are connected to the occipital lobes, are significantly associated with UPSIT scores in PD [20]. Our results could be interpreted in this way, where alterations of some tracts passing through the occipital lobes are associated with olfactory dysfunction. However, further studies are required to interpret this result more precisely. MDS-UPDRS III and UPSIT scores were significantly correlated with the QAs of almost every brain area, while SCOPAAUT and RBDSQ scores only showed significant correlations with a few brain regions, if any, even though some studies found a significant relationship between autonomic or sleep disorders and WM connectivity in PD [49,50]. These findings suggest that motor deficits and olfactory dysfunction could be typical biomarkers that appear earlier than autonomic or sleep disorders and could be correlated with widespread microstructural alterations of the brain in early PD.

Although we conducted this research with careful considerations, there are some limitations. The participants were only recruited from the PPMI database, and the number of participants used for this study may limit the generalizability of this study. It might be useful to explore whether the results of this study are reproducible in further studies. There also may be limitations caused by not dividing the PD patients into subgroups based on the severity of the disease. WM alterations can potentially be significantly different based on the severity of the disease within a single early PD group. By comparing PD subgroups, it would be possible to determine whether the severity or duration would be correlated with WM alterations in PD. One limitation regarding our study is the utilization of data using outdated PPMI protocols. According to previous research, conventional DTI scalar measures such as FA and MD are affected by different acquisition factors such as b-value, resolution, and gradient directions [51]. For example, a study showed that a higher number of gradient directions increased FA while reducing MD [52]. Although we could not find any studies investigating how QA values are affected by different DTI acquisition schemes, we predict that QA would show a similar pattern to FA. Another major limitation is the interpretation of the QAs in the occipital lobe. Although we suggested some hypotheses to interpret the QA differences between HC and PD and the correlations between the QAs of the occipital lobe and UPSIT scores, further studies are needed to explain these results more accurately. However, we believe our study is the first to evaluate whole brain structures using an advanced dMRI processing technique to compare QA as a diffusion metric between HC and PD and to correlate the QAs of PD with several neuropsychological 
test scores. These findings could provide valuable evidence for the pathophysiology of PD and the applicability of dMRI processing techniques in PD.

\section{Conclusions}

In this study, we utilized a dMRI processing technique called GQI to compare QA as a diffusion metric between HCs and PD patients throughout the whole brain region. We were able to find significant QA differences in not only the typical pathologic areas of PD, such as the basal ganglia and brain stem, but also in less-studied areas such as the limbic system, temporal lobe, and occipital lobe. Higher QA values in PD suggest that neural compensatory mechanisms occur in early-stage PD, similar to the results of previous studies on prodromal or early-stage PD. In addition to these results, we found that some subcortical structures such as the basal ganglia, cerebellum, and brain stem are highly correlated with motor dysfunction in PD, while some cortical structures such as the temporal lobe and occipital lobe are highly correlated with olfactory dysfunction in PD. We believe that these results could broaden our understanding of PD. Future studies on PD will be necessary to reproduce and verify our findings.

Author Contributions: Conceptualization, J.-Y.K. and H.-M.B.; methodology, J.-H.S.; software, J.-H.S.; validation, J.-Y.K., H.-M.B. and J.-H.S.; formal analysis, J.-Y.K.; investigation, J.-Y.K.; resources, J.-Y.K.; data curation, J.-Y.K.; writing—original draft preparation, J.-Y.K.; writing—review and editing, H.-M.B.; visualization, J.-Y.K.; supervision, H.-M.B.; project administration, H.-M.B.; funding acquisition, H.-M.B. All authors have read and agreed to the published version of the manuscript.

Funding: PPMI-a public-private partnership-is funded by the Michael J. Fox Foundation for Parkinson's Research and funding partners, including AbbVie, AcureX THERAPEUTICS, Allergan, Amathus Therapeutics, Avid Radiopharmaceuticals, Bial Biotech, Biogen, BioLegend, Bristol-Myers Squibb, Calico, Celgene, DENALI, 4D pharma pic, GE Healthcare, Genentech, GSK GlaxoSmithKline, GOLUB CAPITAL, Handl Therapeutics, insitro, Jannsen Neuroscience, Lilly, Lunbeck, MERCK, MSD Meso Scale Discovery, Neurocrine, Pfizer, Piramal, Prevail Therapeutics, Roche, Sanofi Genzyme, Servier, Takeda, Teva, UCB, Verily, Voyager Therapeutics.

Institutional Review Board Statement: Not applicable.

Informed Consent Statement: Not applicable.

Data Availability Statement: Data used in the preparation of this article were obtained from the Parkinson's Progression Markers Initiative (PPMI) open-access database (www.ppmi-info.org/data, accessed on 1 March 2021).

Acknowledgments: This research was supported by the Brain Research Program through the National Research Foundation of Korea (NRF) funded by the Ministry of Science and ICT (NRF2017M3C7A1044367), Basic Science Research Capacity Enhancement Project through Korea Basic Science Institute (National research Facilities and Equipment Center) grant funded by the Ministry of Education (grant No. 2021R1A6C101A432), and Bio \& Medical Technology Development Program of the National Research Foundation funded by the Korea government (MSIT) (2020M3A9E4104384).

Conflicts of Interest: The authors declare no conflict of interest.

\section{References}

1. Xia, R.; Mao, Z.H. Progression of motor symptoms in Parkinson's disease. Neurosci. Bull. 2012, 28, 39-48. [CrossRef]

2. Devi, L.; Raghavendran, V.; Prabhu, B.M.; Avadhani, N.G.; Anandatheerthavarada, H.K. Mitochondrial import and accumulation of alpha-synuclein impair complex I in human dopaminergic neuronal cultures and Parkinson disease brain. J. Biol. Chem. 2008, 283, 9089-9100. [CrossRef]

3. Braak, H.; Ghebremedhin, E.; Rüb, U.; Bratzke, H.; Del Tredici, K. Stages in the development of Parkinson's disease-related pathology. Cell Tissue Res. 2004, 318, 121-134. [CrossRef]

4. Salat, D.; Noyce, A.J.; Schrag, A.; Tolosa, E. Challenges of modifying disease progression in prediagnostic Parkinson's disease. Lancet Neurol. 2016, 15, 637-648. [CrossRef]

5. Postuma, R.B.; Aarsland, D.; Barone, P.; Burn, D.J.; Hawkes, C.H.; Oertel, W.; Ziemssen, T. Identifying prodromal Parkinson's disease: Pre-motor disorders in Parkinson's disease. Mov. Disord. 2012, 27, 617-626. [CrossRef] [PubMed] 
6. Atkinson-Clement, C.; Pinto, S.; Eusebio, A.; Coulon, O. Diffusion tensor imaging in Parkinson's disease: Review and metaanalysis. Neuroimage Clin. 2017, 16, 98-110. [CrossRef]

7. Assaf, Y.; Pasternak, O. Diffusion tensor imaging (DTI)-based white matter mapping in brain research: A review. J. Mol. Neurosci. 2008, 34, 51-61. [CrossRef]

8. Salan, T.; Jacobs, E.L.; Reddick, W.E. A 3D model-based simulation of demyelination to understand its effects on diffusion tensor imaging. Annu. Int. Conf. IEEE Eng. Med. Biol. Soc. 2017, 2017, 3525-3528.

9. $\mathrm{Gu}, \mathrm{L} . ; \mathrm{Li}$, J.; Feng, D.F.; Cheng, E.T.; Li, D.C.; Yang, X.Q.; Wang, B.C. Detection of white matter lesions in the acute stage of diffuse axonal injury predicts long-term cognitive impairments: A clinical diffusion tensor imaging study. J. Trauma. Acute Care Surg. 2013, 74, 242-247. [CrossRef]

10. Winklewski, P.J.; Sabisz, A.; Naumczyk, P.; Jodzio, K.; Szurowska, E.; Szarmach, A. Understanding the Physiopathology Behind Axial and Radial Diffusivity Changes-What Do We Know? Front. Neurol. 2018, 9, 92. [CrossRef]

11. Yeh, F.C.; Wedeen, V.J.; Tseng, W.Y. Generalized q-sampling imaging. IEEE Trans. Med. Imaging 2010, 29, 1626-1635.

12. Jin, Z.; Bao, Y.; Wang, Y.; Li, Z.; Zheng, X.; Long, S.; Wang, Y. Differences between generalized Q-sampling imaging and diffusion tensor imaging in visualization of crossing neural fibers in the brain. Surg. Radiol. Anat. 2019, 41, 1019-1028. [CrossRef]

13. Shen, C.Y.; Tyan, Y.S.; Kuo, L.W.; Wu, C.W.; Weng, J.C. Quantitative Evaluation of Rabbit Brain Injury after Cerebral Hemisphere Radiation Exposure Using Generalized q-Sampling Imaging. PLoS ONE 2015, 10, e0133001. [CrossRef]

14. Celtikci, P.; Fernandes-Cabral, D.T.; Yeh, F.C.; Panesar, S.S.; Fernandez-Miranda, J.C. Generalized q-sampling imaging fiber tractography reveals displacement and infiltration of fiber tracts in low-grade gliomas. Neuroradiology 2018, 60, 267-280. [CrossRef]

15. Schwarz, S.T.; Abaei, M.; Gontu, V.; Morgan, P.S.; Bajaj, N.; Auer, D.P. Diffusion tensor imaging of nigral degeneration in Parkinson's disease: A region-of-interest and voxel-based study at $3 \mathrm{~T}$ and systematic review with meta-analysis. Neuroimage Clin. 2013, 3, 481-488. [CrossRef]

16. Nagae, L.M.; Honce, J.M.; Tanabe, J.; Shelton, E.; Sillau, S.H.; Berman, B.D. Microstructural Changes within the Basal Ganglia Differ between Parkinson Disease Subtypes. Front. Neuroanat. 2016, 10, 17. [CrossRef]

17. Chen, N.K.; Chou, Y.H.; Sundman, M.; Hickey, P.; Kasoff, W.S.; Bernstein, A.; Trouard, T.P.; Lin, T.; Rapcsak, S.Z.; Sherman, S.J.; et al. Alteration of Diffusion-Tensor Magnetic Resonance Imaging Measures in Brain Regions Involved in Early Stages of Parkinson's Disease. Brain Connect. 2018, 8, 343-349. [CrossRef]

18. Wen, M.C.; Heng, H.S.E.; Hsu, J.L.; Xu, Z.; Liew, G.M.; Au, W.L.; Chan, L.L.; Tan, L.C.S.; Tan, E.K. Structural connectome alterations in prodromal and de novo Parkinson's disease patients. Parkinsonism Relat. Disord. 2017, 45, 21-27. [CrossRef]

19. Mishra, V.R.; Sreenivasan, K.R.; Yang, Z.; Zhuang, X.; Cordes, D.; Mari, Z.; Litvan, I.; Fernandez, H.H.; Eidelberg, D.; Ritter, A.; et al. Unique white matter structural connectivity in early-stage drug-naive Parkinson disease. Neurology 2020, 94, e774-e784. [CrossRef]

20. Sobhani, S.; Rahmani, F.; Aarabi, M.H.; Sadr, A.V. Exploring white matter microstructure and olfaction dysfunction in early parkinson disease: Diffusion MRI reveals new insight. Brain Imaging Behav. 2019, 13, 210-219. [CrossRef]

21. Vaillancourt, D.E.; Spraker, M.B.; Prodoehl, J.; Abraham, I.; Corcos, D.M.; Zhou, X.J.; Comella, C.L.; Little, D.M. High-resolution diffusion tensor imaging in the substantia nigra of de novo Parkinson disease. Neurology 2009, 72, 1378-1384. [CrossRef] [PubMed]

22. Pelizzari, L.; Laganà, M.M.; Di Tella, S.; Rossetto, F.; Bergsland, N.; Nemni, R.; Clerici, M.; Baglio, F. Combined Assessment of Diffusion Parameters and Cerebral Blood Flow Within Basal Ganglia in Early Parkinson's Disease. Front. Aging Neurosci. 2019, 11, 134. [CrossRef] [PubMed]

23. Mangia, S.; Svatkova, A.; Mascali, D.; Nissi, M.J.; Burton, P.C.; Bednarik, P.; Auerbach, E.J.; Giove, F.; Eberly, L.E.; Howell, M.J.; et al. Multi-modal Brain MRI in Subjects with PD and iRBD. Front. Neurosci. 2017, 11, 709. [CrossRef] [PubMed]

24. Mole, J.P.; Subramanian, L.; Bracht, T.; Morris, H.; Metzler-Baddeley, C.; Linden, D.E. Increased fractional anisotropy in the motor tracts of Parkinson's disease suggests compensatory neuroplasticity or selective neurodegeneration. Eur. Radiol. 2016, 26, 3327-3335. [CrossRef]

25. Wen, M.C.; Heng, H.S.; Ng, S.Y.; Tan, L.C.; Chan, L.L.; Tan, E.K. White matter microstructural characteristics in newly diagnosed Parkinson's disease: An unbiased whole-brain study. Sci. Rep. 2016, 6, 35601. [CrossRef]

26. Bergamino, M.; Keeling, E.G.; Mishra, V.R.; Stokes, A.M.; Walsh, R.R. Assessing White Matter Pathology in Early-Stage Parkinson Disease Using Diffusion MRI: A Systematic Review. Front. Neurol. 2020, 11, 314. [CrossRef]

27. Andersson, J.L.R.; Sotiropoulos, S.N. An integrated approach to correction for off-resonance effects and subject movement in diffusion MR imaging. Neuroimage 2016, 125, 1063-1078. [CrossRef]

28. Tournier, J.D.; Smith, R.; Raffelt, D.; Tabbara, R.; Dhollander, T.; Pietsch, M.; Christiaens, D.; Jeurissen, B.; Yeh, C.H.; Connelly, A. MRtrix3: A fast, flexible and open software framework for medical image processing and visualisation. Neuroimage 2019, 202, 116137. [CrossRef]

29. Desikan, R.S.; Ségonne, F.; Fischl, B.; Quinn, B.T.; Dickerson, B.C.; Blacker, D.; Buckner, R.L.; Dale, A.M.; Maguire, R.P.; Hyman, B.T.; et al. An automated labeling system for subdividing the human cerebral cortex on MRI scans into gyral based regions of interest. Neuroimage 2006, 31, 968-980. [CrossRef]

30. Sveinbjornsdottir, S. The clinical symptoms of Parkinson's disease. J. Neurochem. 2016, 139, 318-324. [CrossRef]

31. Wei, X.; Yan, R.; Chen, Z.; Weng, R.; Liu, X.; Gao, H.; Xu, X.; Kang, Z.; Liu, Z.; Guo, Y.; et al. Combined Diffusion Tensor Imaging and Arterial Spin Labeling as Markers of Early Parkinson's disease. Sci. Rep. 2016, 6, 33762. [CrossRef] 
32. Joshi, N.; Rolheiser, T.M.; Fisk, J.D.; McKelvey, J.R.; Schoffer, K.; Phillips, G.; Armstrong, M.; Khan, M.N.; Leslie, R.A.; Rusak, B.; et al. Lateralized microstructural changes in early-stage Parkinson's disease in anterior olfactory structures, but not in substantia nigra. J. Neurol. 2017, 264, 1497-1505. [CrossRef] [PubMed]

33. Galvan, A.; Devergnas, A.; Wichmann, T. Alterations in neuronal activity in basal ganglia-thalamocortical circuits in the parkinsonian state. Front. Neuroanat. 2015, 9, 5. [CrossRef] [PubMed]

34. Skidmore, F.M.; Spetsieris, P.G.; Anthony, T.; Cutter, G.R.; von Deneen, K.M.; Liu, Y.; White, K.D.; Heilman, K.M.; Myers, J.; Standaert, D.G.; et al. A full-brain, bootstrapped analysis of diffusion tensor imaging robustly differentiates Parkinson disease from healthy controls. Neuroinformatics 2015, 13, 7-18. [CrossRef]

35. Sanjari Moghaddam, H.; Dolatshahi, M.; Salardini, E.; Aarabi, M.H. Association of olfaction dysfunction with brain microstructure in prodromal Parkinson disease. Neurol. Sci. 2019, 40, 283-291. [CrossRef]

36. Gregory, S.; Long, J.D.; Klöppel, S.; Razi, A.; Scheller, E.; Minkova, L.; Papoutsi, M.; Mills, J.A.; Durr, A.; Leavitt, B.R.; et al Operationalizing compensation over time in neurodegenerative disease. Brain 2017, 140, 1158-1165. [CrossRef] [PubMed]

37. Fullard, M.E.; Morley, J.F.; Duda, J.E. Olfactory Dysfunction as an Early Biomarker in Parkinson's Disease. Neurosci. Bull. 2017, 33, 515-525. [CrossRef]

38. Zald, D.H.; Pardo, J.V. Emotion, olfaction, and the human amygdala: Amygdala activation during aversive olfactory stimulation Proc. Natl. Acad. Sci. USA 1997, 94, 4119-4124. [CrossRef]

39. Kay, L.M.; Freeman, W.J. Bidirectional processing in the olfactory-limbic axis during olfactory behavior. Behav. Neurosci. 1998, 112, 541-553. [CrossRef]

40. Ibarretxe-Bilbao, N.; Junque, C.; Marti, M.J.; Valldeoriola, F.; Vendrell, P.; Bargallo, N.; Zarei, M.; Tolosa, E. Olfactory impairment in Parkinson's disease and white matter abnormalities in central olfactory areas: A voxel-based diffusion tensor imaging study. Mov. Disord. 2010, 25, 1888-1894. [CrossRef]

41. Roy-Côté, F.; Zahal, R.; Frasnelli, J.; Nguyen, D.K.; Boucher, O. Insula and Olfaction: A Literature Review and Case Report. Brain Sci. 2021, 11, 198. [CrossRef] [PubMed]

42. Arrigo, A.; Calamuneri, A.; Milardi, D.; Mormina, E.; Rania, L.; Postorino, E.; Marino, S.; Di Lorenzo, G.; Anastasi, G.P.; Ghilardi, M.F.; et al. Visual System Involvement in Patients with Newly Diagnosed Parkinson Disease. Radiology 2017, 285, 885-895. [CrossRef] [PubMed]

43. Armstrong, R.A. Visual Dysfunction in Parkinson's Disease. Int. Rev. Neurobiol. 2017, 134, 921-946. [PubMed]

44. Guimarães, R.P.; Campos, B.M.; de Rezende, T.J.; Piovesana, L.; Azevedo, P.C.; Amato-Filho, A.C.; Cendes, F.; D’Abreu, A. Is Diffusion Tensor Imaging a Good Biomarker for Early Parkinson's Disease? Front. Neurol. 2018, 9, 626. [CrossRef] [PubMed]

45. Zhang, Y.; Wu, I.W.; Buckley, S.; Coffey, C.S.; Foster, E.; Mendick, S.; Seibyl, J.; Schuff, N. Diffusion tensor imaging of the nigrostriatal fibers in Parkinson's disease. Mov. Disord. 2015, 30, 1229-1236. [CrossRef] [PubMed]

46. Rolheiser, T.M.; Fulton, H.G.; Good, K.P.; Fisk, J.D.; McKelvey, J.R.; Scherfler, C.; Khan, N.M.; Leslie, R.A.; Robertson, H.A Diffusion tensor imaging and olfactory identification testing in early-stage Parkinson's disease. J. Neurol. 2011, 258, 1254-1260. [CrossRef] [PubMed]

47. Bohnen, N.I.; Müller, M.L. In vivo neurochemical imaging of olfactory dysfunction in Parkinson's disease. J. Neural. Transm. 2013, 120, 571-576. [CrossRef]

48. Braak, H.; Del Tredici, K.; Rüb, U.; de Vos, R.A.; Jansen Steur, E.N.; Braak, E. Staging of brain pathology related to sporadic Parkinson's disease. Neurobiol. Aging. 2003, 24, 197-211. [CrossRef]

49. Ansari, M.; Rahmani, F.; Dolatshahi, M.; Pooyan, A.; Aarabi, M.H. Brain pathway differences between Parkinson's disease patients with and without REM sleep behavior disorder. Sleep Breath. 2017, 21, 155-161. [CrossRef]

50. Ashraf-Ganjouei, A.; Majd, A.; Javinani, A.; Aarabi, M.H. Autonomic dysfunction and white matter microstructural changes in drug-naïve patients with Parkinson's disease. PeerJ 2018, 6, e5539. [CrossRef]

51. Barrio-Arranz, G.; de Luis-Garcia, R.; Tristan-Vega, A.; Martin-Femandez, M.; Aja-Fernandez, S. Impact of MR Acquisition Parameters on DTI Scalar Indexes: A Tractography Based Approach. PLoS ONE 2015, 10, e0137905. [CrossRef] [PubMed]

52. Wang, J.; Abdi, H.; Bakhadirov, K.; Diaz-Arrastia, R.; Devous, M. A comprehensive reliability assessment of quantitative diffusion tensor tractography. Neuroimage 2012, 60, 1127-1138. [CrossRef] [PubMed] 